\title{
Estimativa da resistência característica à tração da madeira na direção paralela às fibras por meio de modelos probabilísticos
}

\section{Estimation of the characteristic tensile strength of the wood in the parallel direction to the grains through of probability models}

Anderson Renato Vobornik Wolenski ${ }^{1}$, Rodrigo Guerra Peixoto ${ }^{2}$, André Luís Christoforo ${ }^{3}$, Francisco Antonio Rocco Lahr ${ }^{4}$, Alfredo Manuel Pereira Geraldes Dias ${ }^{5}$,

\footnotetext{
${ }^{1}$ Laboratório de Construção Civil - Instituto Federal de Santa Catarina (IFSC) - Av. Aloísio Stoffel, 1271, Jardim Alvorada, CEP: 89885-000, São Carlos, SC, Brasil.

${ }^{2}$ Programa de Pós-graduação em Engenharia de Estruturas (PROPEEs) - UFMG - Av. Antonio Carlos, 6627, Pampulha, CEP: 31270-901, Belo Horizonte, MG, Brasil.

${ }^{3}$ Programa de Pós-graduação em Estruturas e Construção Civil (PPGECiv) - UFSCAr - Rodovia Washington Luís, s/n, CEP: 13565-905, São Carlos, SP, Brasil.

${ }^{4}$ Laboratório de Madeiras e Estruturas de Madeiras (LaMEM) - USP - Av. Trabalhador Sãocarlense, 400, CEP: 13566590, São Carlos, SP, Brasil.

${ }^{5}$ Departamento de Engenharia Civil da Universidade de Coimbra (UC) - Rua Luís Reis Santos - Pólo II, CEP: 3030-788, Coimbra, Beira Litoral, Portugal.

e-mail: anderson.wolenski@ifsc.edu.br, rodrigo.peixoto@dees.ufmg.br, christoforoal@yahoo.com.br, frocco@sc.usp.br, alfgdias@dec.uc.pt
}

\section{RESUMO}

Para um dimensionamento de estruturas de madeiras dentro das exigências de segurança, a resistência para determinada solicitação mecânica é calculada com base em seu valor característico, correspondente ao percentil de 5\% de um determinado modelo de distribuição de probabilidades. A norma brasileira NBR 7190 [1] estabelece, para pequenas amostras, relações para estimar a resistência característica da madeira, que podem resultar em diferença significativa dos valores obtidos quando comparado a um modelo probabilístico adequado. Considerando os resultados da resistência à tração $\left(f_{\mathrm{t} 0, \mathrm{k}}\right)$ na direção paralela às fibras para 40 distintas espécies de madeira folhosas, com 480 determinações experimentais com teor de umidade padrão $(\approx 12 \%)$, empregou-se funções densidade de distribuição de probabilidades (Normal, LogNormal, Weibull e Exponencial) para cada espécie visando determinar o modelo de maior aderência. Uma vez determinados os valores e os respectivos modelos para cada espécie, um modelo de regressão linear multivariável, fundamentado na análise de variância (ANOVA) e dependentes do valor médio $(\bar{x})$, do coeficiente de variação (CV\%) e do menor $(\min )$ e maior $(\max )$ valor da resistência para cada espécie, é adotado com objetivo de estimar a resistência $f_{\mathrm{t} 0, \mathrm{k}}$ ajustada com aqueles termos mais significativos, de modo a inferir na qualidade do estimador e, consequentemente, na confiabilidade da obtenção desta propriedade mecânica. Por fim, o modelo de regressão aqui proposto é comparado a proposta da norma brasileira, a fim de avaliar a confiabilidade do modelo e sua adequação na estimativa da resistência $f_{\mathrm{t} 0, \mathrm{k}}$.

Palavras-chave: madeiras tropicais, propriedades de resistência, valor característico.

\section{ABSTRACT}

For a structural design of wood within of the safety requirements, the strength for a certain mechanical request is calculated based on its characteristic value, corresponding to the $5 \%$ percentile of a given probability 
distribution model. The Brazilian standard NBR 7190 [1] establishes, for small samples, relations to estimate the characteristic strength of the wood, which can result in a significant difference of the values obtained when compared to a suitable probabilistic model. Considering the results of the tensile strength $\left(f_{\mathrm{t} 0, \mathrm{k}}\right)$ in the direction parallel to the grain for 40 different hardwood species, with 480 experimental determinations with standard moisture content $(\approx 12 \%$ ), were used distribution density functions (Normal, LogNormal, Weibull and Exponential) for each species, in order to determine the highest adhesion model. Once determined the values and respective models for each species, a multivariable linear regression model, based on analysis of variance (ANOVA) and dependent on the mean value $(\bar{x})$, coefficient of variation $(\mathrm{CV} \%)$ and lowest $(\mathrm{min})$ and the highest ( $\max$ ) strength value for each species, is adopted to estimate the $f_{\mathrm{t} 0, \mathrm{k}}$ adjusted with the most significant terms, in order to infer the quality of the estimator and, consequently, the reliability of such mechanical property. Finally, the regression model proposed here is compared to the proposition of the Brazilian standard, in order to evaluate the reliability of the model and its adequacy in the estimation of the tensile strength $f_{\mathrm{t} 0, \mathrm{k}}$ in the direction parallel to the grain.

Keywords: tropical woods, strength properties, characteristic value.

\section{INTRODUÇÃO}

A madeira, como elemento estrutural, possui elevado potencial de aplicabilidade no Brasil, proporcionado pela numerosa quantidade de espécies existentes na Floresta Amazônica. Segundo STEEGE et al.[2], até 2015, já haviam sido catalogadas mais de 10 mil espécies. Este número expressivo corrobora para o desenvolvimento de novas pesquisas visando à caracterização de espécies com potencial substitutivo àquelas comumente adotadas na construção civil. Em destaque, pode-se citar trabalhos originados por da SILVA et al.[3], CARREIRA et al.[4], ALMEIDA et al.[5], CHRISTOFORO et al.[6], LAHR et al.[7], dos REIS et al.[8], entre outros, que buscaram caracterizar novas espécies para fins estruturais.

No Brasil, quem regulamenta o uso de madeiras para esta finalidade é a NBR 7190 [1]. Dentre seus principais pontos, ela estabelece os condicionantes para elaboração de projetos e as exigências para execução e controle de estruturas de madeira, sendo pautada em métodos probabilísticos como na verificação do estado limite último, que avalia a capacidade resistente à ruptura e a instabilidade da estrutura, e na verificação do estado limite de utilização, responsável por avaliar a deformação excessiva e a durabilidade da estrutura.

Tais métodos supõem-se uma distribuição normal dos valores das resistências às solicitações mecânicas das madeiras, sendo esta a favor da segurança, ao condicionar tais valores a um valor característico correspondente ao percentil de $5 \%$ da respectiva distribuição de resistências, em conjunto com um valor limite para o coeficiente de variação igual a $\delta=18 \%$. Logsdon et al. [9] afirma, contudo, que tal critério pode não ser uma regra única na obtenção dos valores característicos das solicitações mecânicas. No trabalho em questão, os autores buscaram aferir o modelo para estimar a resistência característica à compressão $f_{\mathrm{c} 0 \mathrm{k}, \mathrm{k}}$, objetivando um modelo estatístico mais adequado para a espécie Angelim Pedra, concluindo que as equações da referida norma são mais conservadoras ao fornecerem valores levemente menores para a $f_{\mathrm{c} 0, \mathrm{k}}$.

Analogamente ao estudo anterior, Matos e Molina [10] estudaram a relação entre as resistências à compressão e ao cisalhamento $\left(f_{\mathrm{V} 0, \mathrm{k}} / f_{\mathrm{c} 0, \mathrm{k}}\right)$ das espécies de madeira de Pinus elliotti e Eucalyptus citriodora (Corymbia citriodora), comparando a relação obtida experimentalmente com as relações constantes nas normativas NBR 7190 [1] e ISO 13910 [11] e concluíram que as relações obtidas pela norma brasileira, para ambas às espécies, foram superiores às relações da normativa europeia.

Por outro lado, Pinto et al. [12] e Espinosa et al. [13] avaliaram resultados experimentais para 100 corpos de prova da espécie do gênero Eucalyptus grandis, visando obter a resistência $f_{\mathrm{c} 0, \mathrm{k}}$ a partir do método probabilístico da norma brasileira [1], comparando-o ao método da teoria dos percentis para dados agrupados. Em ambos os trabalhos pôde-se concluir que a equação dos percentis se apresentou como a mais adequada para distribuições assimétricas (assimetria comumente encontrada na amostragem experimental), já que o valor obtido pela metodologia da norma apresentou um erro não conservador na ordem de $12 \%$.

Enquanto os estudos anteriores se restringiram a análise de poucas espécies, a proposta de Dias e Lahr [14] reporta a caracterização de 40 espécies de madeira nativas, visando avaliar as equações simplificadas da norma brasileira [1] quanto à obtenção dos valores característicos para as resistências mecânicas, concluindo que as relações para as resistências ao cisalhamento e compressão da norma não se mostraram equivalentes ao conjunto obtido pela amostragem experimental. Destaca-se que dentre o conjunto de espécies estudadas no referido trabalho, 32 espécies também são aqui avaliadas.

Em comum, tais estudos demonstram a importância da obtenção das propriedades mecânicas, visando alcançar estimativas razoáveis e seguras para o dimensionamento de estruturas de madeira. Por isso, é notá- 
vel a relevância por trabalhos que objetivem a obtenção de equações estimadoras das propriedades mecânicas para distintas espécies de uso estrutural, assim como prediz a norma NBR 7190 [1] ao propor um método probabilístico para obtenção das diferentes propriedades mecânicas das madeiras.

A necessidade por tais estimativas, portando, conduz este trabalho na avaliação de 40 espécies de madeira pertencentes ao grupo das folhosas. Quando comparado a NBR 7190 [1] (p. 90, Anexo E), a mesma traz valores médios para as propriedades físico-mecânicas de 43 espécies de madeiras nativas e de reflorestamento, conjunto este muito próximo às espécies aqui avaliadas, o que reforça o grau de confiabilidade das análises estatísticas aqui conduzidas exclusivamente para madeiras de florestas nativas.

Assim, com o uso de modelos de distribuição de probabilidades, este trabalho objetiva estimar o valor característico à tração $\left(f_{\mathrm{t} 0, \mathrm{k}}\right)$ na direção paralela às fibras para um conjunto de 40 espécies de madeira do grupo das folhosas, associando tal valor ao modelo probabilístico mais adequando, dentre os modelos Normal, LogNormal, Weibull e Exponencial. Na sequência, o conjunto formado pelos valores característicos de cada espécie será relacionado com um modelo de regressão linear multivariável, fundamentado na análise de variância (ANOVA) e dependente do valor médio $(\bar{x})$, do coeficiente de variação (CV\%) e do menor $(\min )$ e maior $(\max )$ valor da $f_{\mathrm{t} 0 \mathrm{k},}$, a fim de ajustar tal resistência com os termos mais significativos, inferindo na qualidade da estimativa na confiabilidade dessa propriedade mecânica. Por fim, uma comparação desta regressão com a equação empírica da norma brasileira é realizada para avaliar a adequação destes modelos na obtenção desta propriedade visando sua segura adoção para o dimensionamento estrutural.

\section{MATERIAIS E MÉTODOS}

Os valores da resistência $f_{\mathrm{t} 0 \mathrm{k}, \mathrm{k}}$ para as 40 espécies aqui estudadas, foram obtidos seguindo as premissas e métodos de ensaio e de cálculo da NBR 7190 [1] (p. 47, Anexo B). Os equacionamentos para avaliar tal propriedade são sumarizados na sequência. Além disto, são detalhados os modelos para as distribuições de probabilidades aqui consideradas, assim como o modelo de regressão linear multivariável, fundamentado na análise ANOVA para estimar a resistência $f_{\mathrm{t} 0, \mathrm{k}}$, considerando o conjunto de resultados das 40 espécies.

Os ensaios foram realizados no Laboratório de Madeiras e de Estruturas de Madeira (LaMEM), da Escola de Engenharia de São Carlos, Universidade de São Paulo (USP). Para a resistência $f_{\text {to }}$ foram preparados e ensaiados 12 corpos de prova para cada uma das 40 espécies apresentadas na Tabela 1, resultando um total de $12 \times 40=480$ determinações experimentais.

Tabela 1: Nomes e identificação (ID) das 40 espécies nativas do grupo das folhosas.

\begin{tabular}{|c|c|c|c|c|c|}
\hline ID & POPULAR & CIENTÍFICO & ID & POPULAR & CIENTÍFICO \\
\hline 01 & Angelim-amargoso & Vatairea fusca & 21 & Cutiúba & Goupia paraensis \\
\hline 02 & Angelim-araroba & Vataireopsis araroba & 22 & Garapa & Apuleia leiocarpa \\
\hline 03 & Angelim-ferro & Hymenolobium cf. heterocarpum & 23 & Goiabão & Planchonella pachycarpa \\
\hline 04 & Angelim-pedra & Hymenolobium petraeum & 24 & Guaiçara & Luetzelburgia cf. guaissara \\
\hline 05 & Angelim-saia & Vatairea cf. guianensis & 25 & Guajará & Micropholis venulosa \\
\hline 06 & Angelim-vermelho & Dinizia excelsa & 26 & Guarucaia & Peltophorum dubium \\
\hline 07 & Angico-branco & Parkia cf. pendula & 27 & Itaúba & Mezilaurus itauba \\
\hline 08 & Angico-preto & Anadenanthera colubrina & 28 & Jatobá & $\overline{\text { Hymenaea courbaril }}$ \\
\hline 09 & Branquilho & Sebastiania commersoniana & 29 & Louro-preto & Ocotea neesiana \\
\hline 10 & Cafearana & Andira anthelmia & 30 & Louro-verde & $\overline{\text { Sextonia cf. rubra }}$ \\
\hline 11 & Cambará-rosa & Erisma cf. fuscum & 31 & Maçaranduba & Manilkara cf. inundata \\
\hline 12 & Canafístula & $\overline{\text { Cassia ferruginea }}$ & 32 & Mandioqueira & Qualea paraensis \\
\hline 13 & Castanheira & $\overline{\text { Bertholletia excelsa }}$ & 33 & Oiticica-amarela & Clarisia racemosa \\
\hline 14 & Castelo & Calycophyllum multiflorum & 34 & Oiuchu & Pradosia sp. \\
\hline 15 & Catanudo & Calophyllum longifolium & 35 & Parinari & Parinari excelsa \\
\hline 16 & Cedro-amargo & Cedrela odorata & 36 & Pau-óleo & Copaifera langsdorffii \\
\hline 17 & Cedro-doce & $\overline{\text { Cedrela } \mathrm{cf} \text {. fissilis }}$ & 37 & Piolho & Tapirira $\mathrm{sp}$. \\
\hline 18 & Cedroarana & Cedreling a cateniformis & 38 & Quarubarana & Erisma uncinatum \\
\hline 19 & Champanhe & $\underline{\text { Dipteryx odorata }}$ & 39 & Quina-rosa & Geissospermum sericeum \\
\hline 20 & Copaíba & Copaifera multijuga & 40 & Rabo-de-arraia & Vochysia haenkeana \\
\hline
\end{tabular}

\subsection{Valores característicos}

As propriedades mecânicas de resistência tiveram seus valores corrigidos para o teor de umidade de $12 \%$, assim como prediz a norma brasileira NBR 7190 [1] sendo $12 \%$ o teor de umidade de equilíbrio estabelecido por esse documento, conforme expressa a equação: 


$$
f_{12}=f_{\mathrm{U} \%}\left[1+\frac{3(\mathrm{U} \%-12)}{100}\right]
$$

em que $f_{12}$ e $f_{\mathrm{U} \%}$ são, respectivamente, as resistências para a umidade corrigida de $12 \%$ e teor de $\mathrm{U} \%$.

Para a determinação dos valores característicos das resistências à tração das espécies aqui avaliadas, necessárias para compor a resistência de cálculo para uma determinada solicitação em um eventual projeto estrutural, deve-se considerar os valores das resistências às solicitações mecânicas das madeiras obtidos a partir do seu valor característico $\left(f_{\mathrm{w}, \mathrm{k}}\right)$, que corresponde a um percentil de $5 \%$ da distribuição de resistências, e expresso segundo a equação a seguir, sendo $f_{m}$ o valor médio da resistência e $\delta$ o respectivo desvio-padrão.

$$
f_{\mathrm{w}, \mathrm{k}}=f_{\mathrm{m}}[1-1,645 \delta]
$$

Em adição, a NBR 7190 [1] (p. 18, item 6.4.7) exige que os projetos de estruturas de madeira sejam dimensionados sob a hipótese de linearidade cinemática (pequenos deslocamentos), além de exigir resistência à ação das forças atuantes. Assim, a metodologia probabilística da referida norma, supõe a normalidade nas distribuições dos valores da resistência, a favor da segurança, considerando um coeficiente de variação $\delta=18 \%$ para a resistência à tração, tal como expressa:

$$
f_{\mathrm{w}, \mathrm{k}}=f_{\mathrm{m}}[1-1,645 \delta] \approx 0,70 f_{\mathrm{m}}
$$

Por outro lado, para uma investigação direta da resistência, a normativa prevê a empregabilidade da equação sequencial, que estima o valor de $f_{w, k}$ na forma:

$$
f_{\mathrm{w}, \mathrm{k}}=\left[2 \frac{f_{1}+f_{2}+f_{3}+\cdots+f_{\left(\frac{\mathrm{n}}{2}-1\right)}}{\left(\frac{\mathrm{n}}{2}-1\right)}-f_{\left(\frac{\mathrm{n}}{2}\right)}\right] .1,10
$$

em que $f_{n}$ corresponde a $\mathrm{n}$ resultados da resistência à determinada solicitação, devendo ser colocados em ordem crescente $\left(f_{1}<f_{2}<f_{3}<\cdots<f_{\mathrm{n}}\right)$ desprezando-se o maior valor se o número de corpos de prova for ímpar. Ressalta-se que a Equação 4 baseia-se no estimador $z_{b}$ para uma amostra de $2 m$ valores $x_{1}+x_{2}+$ $x_{3}+\cdots+x_{2 m}$, assim como expressa a equação:

$$
z_{b}=\left[2 \frac{x_{1}+x_{2}+x_{3}+\cdots+x_{(m-1)}}{(m-1)}-f_{m}\right]
$$

Contudo, para uma distribuição de extremos centrada no valor característico, a Equação 4 tem um acréscimo de 10\%, o que evita, segundo Logsdon et al.[9], que 50\% das estimativas sejam feitas por valores abaixo da resistência característica. A norma brasileira adota, portanto, como valor de $f_{\mathrm{w}, \mathrm{k}}$ o maior dos valores compreendidos entre: a resistência $f_{1}$, a resistência equivalente a $70 \%$ do valor de $f_{m}$ (Equação 3 ) obtidas pela média das amostras ensaiadas e o valor obtido pela Equação 4 ([1], p. 18). Ainda segundo Pinto et al.[12], a Equação 5 pode resultar em valores significativamente diferentes a partir daqueles associados a uma dada função de probabilidade, tendo em vista a diversidade de funções de probabilidade existentes.

\subsection{Análise Estatística.}

A análise para as 40 espécies de madeiras foram inicialmente pautadas em 04 (quatro) funções para as distribuições de probabilidade, sendo estas adotadas na determinação dos valores característicos da resistência $f_{\mathrm{t} 0, \mathrm{k}}$. Assim, as funções densidade de probabilidade $(\boldsymbol{f})$, sobre a variável aleatória $\boldsymbol{x}$ são expressas conforme as Equações 6, 7, 8 e 9 para as funções Normal, LogNormal, Weibull e Exponencial, respectivamente:

$$
f(x)=\frac{1}{\sqrt{2 \cdot \pi \cdot \sigma^{2}}} \cdot e^{-\frac{1}{2} \cdot\left(\frac{x-\mu}{\sigma}\right)^{2}}, x \in(-\infty, \infty)
$$

em que $\boldsymbol{\sigma}$ é o desvio padrão e $\boldsymbol{\mu}$ a média populacional da função normal;

$$
f(x)=\left\{\begin{array}{cl}
\frac{1}{x \cdot \sigma \cdot \sqrt{2 \cdot \pi}} \cdot e^{-\frac{1}{2} \cdot\left(\frac{\ln (x)-\mu}{\sigma^{2}}\right)^{2}} & , \text { se } x>0 \\
0 & , \text { em caso contrário }
\end{array}\right.
$$

sendo $\boldsymbol{\sigma}$ o desvio padrão e $\boldsymbol{\mu}$ a média populacional logarítmica;

$$
f(x)=\frac{\delta}{\alpha^{\delta}} \cdot x^{\delta-1} \cdot e^{-\left(\frac{x}{\alpha}\right)^{\delta}}, x>0
$$


onde $\boldsymbol{\delta}$ e $\boldsymbol{\alpha}$ são os parâmetros de forma e de escala, respectivamente;

$$
f(x)=\lambda \cdot e^{-\lambda \cdot x}, x>0
$$

em que $\lambda$ é o parâmetro de taxa de distribuição da função exponencial.

Os testes de aderência (ao nível de 95\% de confiabilidade) usados para verificar o melhor modelo de distribuição foram obtidos via Método dos Mínimos Quadrados (MMQ) a partir do Software Minitab ${ }^{\circledR}$ [15]. Ressalta-se que o software em questão dispõe de outras funções que podem ser adotadas para avaliar a aderência de um conjunto de dados amostrais, resultando em um estudo mais amplo capaz de comtemplar outras funções probabilísticas, além das quatro aqui testadas.

Obtidos os valores característicos $f_{\mathrm{t} 0, \mathrm{k}}$ para as 40 espécies, dentre os 04 (quatros) modelos de distribuição de probabilidade (Equações 6 a 9) os resultados são relacionados com o valor médio $(\bar{x})$, o coeficiente de variação $(\mathrm{CV} \%)$ e com o menor $(\min )$ e maior $(\max )$ valor da $f_{\mathrm{t} 0, \mathrm{k}}$, por meio de um modelo de regressão linear multivariável, expresso na equação a seguir (em MPa) e fundamentado na ANOVA, com a qualidade do ajuste avaliada pelo coeficiente de determinação $\left(R_{a j}^{2}\right)$.

$$
f_{\mathrm{t} 0, \mathrm{k}}^{\text {Prob }}=\boldsymbol{\beta}_{0}+\beta_{1} \cdot \bar{x}+\beta_{2} \cdot \mathrm{CV}+\boldsymbol{\beta}_{0} \cdot \min +\beta_{0} \cdot \max +\varepsilon
$$

em que $\beta_{i}$ consistem nos coeficientes ajustados pelo MMQ e $\varepsilon$ é o erro aleatório.

A análise ANOVA, ao nível de $5 \%$ de significância $(\mathrm{p}<0,05)$, foi utilizada para verificar a acurácia da Equação 10. Pelas hipóteses admitidas para a formulação ANOVA do modelo de regressão, $p \geq 0,05$ (probabilidade $\mathrm{P}$ ) implica em se aceitar a hipótese nula $\left(\mathrm{H}_{0}\right)$, ou seja, o modelo ou seus coeficientes não são representativos (variações dos fatores não explicam as variações na variável dependente), e representativos no caso de $\mathrm{p}<0,05$ (hipótese alternativa, $\mathrm{H}_{1}$ ).

Conjuntamente, pode-se testar a normalidade na distribuição dos resíduos da ANOVA com o auxílio do teste de Anderson-Darling [16], em conjunto com o gráfico dos resíduos versus valores ajustados (análise da homogeneidade das variâncias e da independência dos resíduos). Pela formulação do teste, P-valor igual ou superior ao nível de significância (5\%) implica em aceitar a normalidade na distribuição dos resíduos e, em caso contrário, de rejeitar essa hipótese. Portanto, após o teste de aderência, o modelo empírico da norma (Equação 4), pode ser avaliado quanto a sua precisão e comparado ao modelo probabilístico aqui proposto.

\section{RESULTADOS}

Inicialmente, são apresentados na Tabela 2 os valores característicos $f_{\mathrm{t} 0, \mathrm{k}}$ calculados via modelo empírico da norma brasileira [1], conforme Equação 4. Em adição, as classes de resistência (CR), obtidas via resistência característica à compressão $\left(f_{\mathrm{c} 0, \mathrm{k}}\right)$, são apresentados para evidenciar que a distribuição dos valores é assimétrica, ao contemplar todas as 04 (quatro) CR definidas pela norma brasileira [1].

Tabela 2: Resultados da resistência à tração via equação da norma $\left(\boldsymbol{f}_{\mathrm{t} 0, \mathbf{k}}\right)$ e das classes de resistência (CR).

\begin{tabular}{llcc|clcc}
\hline ID & POPULAR & CR & $f_{\text {to,k }}$ & ID & POPULAR & CR & $f_{\text {to,k }}$ \\
\hline 01 & Angelim-amargoso & C40 & 52,56 & 21 & Cutiúba & C40 & 75,22 \\
02 & Angelim-araroba & C40 & 48,63 & 22 & Garapa & C60 & 81,62 \\
03 & Angelim-ferro & C60 & 83,51 & 23 & Goiabão & C40 & 83,54 \\
04 & Angelim-pedra & C40 & 59,27 & 24 & Guaiçara & C40 & 80,59 \\
05 & Angelim-saia & C40 & 67,12 & 25 & Guajará & C60 & 83,43 \\
06 & Angelim-vermelho & C60 & 72,02 & 26 & Guarucaia & C40 & 63,98 \\
07 & Angico-branco & C40 & 53,94 & 27 & Itaúba & C60 & 72,60 \\
08 & Angico-preto & C40 & 76,65 & 28 & Jatobá & C60 & 125,29 \\
09 & Branquilho & C40 & 67,20 & 29 & Louro-preto & C40 & 61,36 \\
10 & Cafearana & C40 & 56,30 & 30 & Louro-verde & C40 & 69,87 \\
11 & Cambará-rosa & C20 & 31,79 & 31 & Maçaranduba & C60 & 109,40 \\
12 & Canafístula & C30 & 59,44 & 32 & Mandioqueira & C60 & 65,13 \\
13 & Castanheira & C30 & 61,95 & 33 & Oiticica-amarela & C60 & 74,23 \\
14 & Castelo & C40 & 86,42 & 34 & Oiuchu & C60 & 85,29 \\
15 & Catanudo & C40 & 48,79 & 35 & Parinari & C40 & 79,35 \\
16 & Cedro-amargo & C30 & 44,04 & 36 & Pau-óleo & C40 & 50,30 \\
17 & Cedro-doce & C20 & 48,55 & 37 & Piolho & C40 & 51,14
\end{tabular}




\begin{tabular}{llll|llll}
18 & Cedroarana & C20 & 50,07 & 38 & Quarubarana & C20 & 40,63 \\
19 & Champanhe & C60 & 87,30 & 39 & Quina-rosa & C60 & 81,65 \\
20 & Copaíba & C40 & 52,67 & 40 & Rabo-de-arraia & C40 & 51,39 \\
\hline
\end{tabular}

Comparativamente, com uso das funções densidade de probabilidade, apresentadas nas Equações 6 a 9 , as resistências $f_{\mathrm{t} 0, \mathrm{k}}^{\text {Prob }}$ podem ser calculadas para cada conjunto de resultados (12 corpos de prova) das 40 espécies de madeira, elegendo a função densidade de melhor aderência pelo maior P-valor encontrado dentre as 4 (Quatro) funções. Em adição, os intervalos de confiança (IC) também são apresentados na Tabela 3.

Tabela 3: Resultado da resistência à tração via funções densidade de probabilidade $\left(\boldsymbol{f}_{\mathbf{t} 0, \mathbf{k}}^{\text {Prob }}\right)$.

\begin{tabular}{ccccc|ccccc}
\hline ID & $f_{\mathrm{t} \text { t, }}^{\text {Prob }}$ & IC & P-valor & MODELO & ID & $f_{\text {to, } \mathrm{k}}^{\text {Prob }}$ & IC & P-valor & MODELO \\
\hline 01 & 46,96 & $(37,33 ; 59,08)$ & 0,668 & LogNormal & 21 & 61,01 & $(46,45 ; 80,41)$ & 0,268 & LogNormal \\
02 & 44,31 & $(35,55 ; 55,22)$ & 0,919 & LogNormal & 22 & 74,32 & $(61,86 ; 89,29)$ & 0,908 & LogNormal \\
03 & 52,28 & $(16,92 ; 87,64)$ & 0,907 & Normal & 23 & 70,53 & $(54,64 ; 91,05)$ & 0,823 & LogNormal \\
04 & 49,71 & $(42,91 ; 57,59)$ & 0,038 & LogNormal & 24 & 78,56 & $(58,41 ; 98,71)$ & 0,341 & Normal \\
05 & 47,72 & $(21,17 ; 74,26)$ & 0,797 & Normal & 25 & 76,13 & $(53,41 ; 98,84)$ & 0,893 & Normal \\
06 & 63,73 & $(50,08 ; 81,11)$ & 0,045 & LogNormal & 26 & 56,77 & $(49,38 ; 65,24)$ & 0,676 & LogNormal \\
07 & 50,73 & $(42,09 ; 59,37)$ & 0,318 & Normal & 27 & 57,41 & $(32,98 ; 81,84)$ & 0,603 & Normal \\
08 & 55,92 & $(26,39 ; 85,45)$ & 0,394 & Normal & 28 & 113,99 & $(86,93 ; 141,04)$ & 0,508 & Normal \\
09 & 61,98 & $(52,11 ; 73,71)$ & 0,423 & LogNormal & 29 & 55,46 & $(47,96 ; 64,12)$ & 0,511 & LogNormal \\
10 & 47,31 & $(36,57 ; 61,19)$ & 0,180 & LogNormal & 30 & 67,47 & $(60,79 ; 74,88)$ & 0,867 & LogNormal \\
11 & 27,17 & $(21,18 ; 34,85)$ & 0,053 & LogNormal & 31 & 102,75 & $(88,48 ; 119,33)$ & 0,334 & LogNormal \\
12 & 51,03 & $(33,15 ; 68,91)$ & 0,297 & Normal & 32 & 45,43 & $(20,31 ; 70,55)$ & 0,796 & Normal \\
13 & 54,80 & $(39,69 ; 75,66)$ & 0,159 & Weibull & 33 & 64,55 & $(53,88 ; 77,34)$ & 0,250 & Weibull \\
14 & 81,49 & $(72,16 ; 92,04)$ & 0,768 & LogNormal & 34 & 75,43 & $(59,72 ; 95,27)$ & 0,848 & LogNormal \\
15 & 37,88 & $(28,85 ; 49,75)$ & 0,083 & LogNormal & 35 & 56,88 & $(27,08 ; 86,68)$ & 0,935 & Normal \\
16 & 38,84 & $(31,83 ; 47,40)$ & 0,347 & LogNormal & 36 & 46,27 & $(37,93 ; 56,45)$ & 0,292 & LogNormal \\
17 & 45,91 & $(38,01 ; 55,45)$ & 0,764 & LogNormal & 37 & 40,05 & $(29,99 ; 53,47)$ & 0,214 & LogNormal \\
18 & 33,31 & $(22,76 ; 48,77)$ & 0,038 & Weibull & 38 & 37,40 & $(26,51 ; 48,29)$ & 0,527 & Normal \\
19 & 85,42 & $(71,09 ; 102,65)$ & 0,754 & LogNormal & 39 & 72,37 & $(47,98 ; 96,76)$ & 0,900 & Normal \\
20 & 48,82 & $(40,53 ; 58,81)$ & 0,227 & LogNormal & 40 & 45,63 & $(36,77 ; 56,63)$ & 0,319 & LogNormal \\
\hline
\end{tabular}

Das 40 espécies avaliadas 32,5\% (13/40) obtiveram melhores ajustes pelo modelo de distribuição Normal, 60\% (24/40) ajustadas pelo modelo LogNormal e 7,5\% (3/40) pelo modelo de Weibull e com o modelo Exponencial não fornecendo ajuste significativo para nenhuma das espécies avaliadas.

Uma vez eleitos os modelos de densidade para as 40 espécies, os valores médios ( $\bar{x}$ em MPa), o coeficiente de variação $(C V \%)$ e os menores $(\min )$ e maiores (max) valores da resistência $f_{\text {to }}$, são apresentados na Tabela 4, visando adotá-los na construção da equação para estimar a resistência $f_{\mathrm{t} 0, \mathrm{k}}^{\mathrm{Mod}}$, conforme modelo de regressão apresentado na Equação 10. 
Tabela 4: Resultados estatísticos obtidos para a resistência $\boldsymbol{f}_{\text {t0 }}$ (em MPa) das 40 espécies de madeira.

\begin{tabular}{ccccc|ccccc}
\hline ID & $\overline{\boldsymbol{x}}$ & $\mathbf{C V} \%$ & $\min$ & $\max$ & $\mathbf{I D}$ & $\overline{\boldsymbol{x}}$ & $\mathbf{C V} \%$ & $\min$ & $\max$ \\
\hline 01 & 75,08 & 26,71 & 45,00 & 105,00 & 21 & 107,50 & 32,81 & 66,20 & 165,60 \\
02 & 69,48 & 27,07 & 42,70 & 108,00 & 22 & 107,64 & 22,50 & 72,40 & 152,60 \\
03 & 119,30 & 35,67 & 60,20 & 197,00 & 23 & 119,30 & 29,70 & 65,70 & 178,60 \\
04 & 66,66 & 17,95 & 47,30 & 86,70 & 24 & 115,13 & 20,25 & 69,30 & 153,60 \\
05 & 95,88 & 32,03 & 33,30 & 152,00 & 25 & 119,18 & 22,94 & 64,50 & 160,20 \\
06 & 104,90 & 32,96 & 58,80 & 198,00 & 26 & 74,88 & 17,26 & 54,30 & 103,40 \\
07 & 67,10 & 15,49 & 53,94 & 84,86 & 27 & 103,72 & 28,35 & 56,30 & 154,00 \\
08 & 109,5 & 31,20 & 51,00 & 161,30 & 28 & 165,27 & 19,70 & 103,30 & 206,00 \\
09 & 87,88 & 21,78 & 67,20 & 124,00 & 29 & 74,07 & 17,92 & 58,80 & 100,20 \\
10 & 80,42 & 29,66 & 45,00 & 111,10 & 30 & 82,80 & 12,55 & 65,480 & 104,47 \\
11 & 45,41 & 30,39 & 30,60 & 66,70 & 31 & 138,48 & 18,16 & 109,40 & 174,80 \\
12 & 84,92 & 25,34 & 40,00 & 109,20 & 32 & 93,04 & 32,49 & 40,70 & 140,00 \\
13 & 88,50 & 24,56 & 54,00 & 118,00 & 33 & 85,83 & 14,94 & 53,30 & 106,50 \\
14 & 103,64 & 14,58 & 80,10 & 125,30 & 34 & 121,80 & 29,25 & 80,20 & 201,60 \\
15 & 65,37 & 38,07 & 45,90 & 129,50 & 35 & 113,40 & 31,64 & 53,80 & 169,10 \\
16 & 58,20 & 24,91 & 37,10 & 88,00 & 36 & 69,29 & 25,11 & 50,30 & 101,20 \\
17 & 67,23 & 22,80 & 44,40 & 97,70 & 37 & 73,05 & 32,76 & 40,40 & 102,30 \\
18 & 61,85 & 26,89 & 46,30 & 93,10 & 38 & 58,05 & 22,58 & 32,70 & 76,40 \\
19 & 121,70 & 21,57 & 87,30 & 173,30 & 39 & 116,64 & 24,20 & 77.00 & 171,00 \\
20 & 71,13 & 23,11 & 50,30 & 101,20 & 40 & 70,94 & 27,49 & 48,40 & 110,10 \\
\hline
\end{tabular}

Assim, o modelo de regressão para a estimativa do valor característico $f_{\mathrm{t} 0, \mathrm{k}}^{\mathrm{Mod}}$ pode ser expresso pela Equação 11, com os resultados da ANOVA apresentados na Tabela 5.

$$
f_{\mathrm{t} 0, \mathrm{k}}^{\mathrm{Mod}}=20,05+0,415 \cdot \bar{x}-0,814 \cdot \mathrm{CV}+0,371 \cdot \min -0,0045 \cdot \max \quad\left[\mathrm{R}_{\mathrm{aj}}^{2}=96,97 \%\right]
$$

Tabela 5: Resultados da ANOVA para o modelo de regressão de acordo com a Equação 11.

\begin{tabular}{lccccc}
\hline \multicolumn{1}{c}{ FATOR } & $\mathrm{GL}$ & $\mathrm{SQ}_{\mathrm{aj}}$ & $\mathrm{QM}_{\mathrm{aj}}$ & VALOR F & P-valor \\
\hline $\bar{x}$ & 1 & 289,0 & 288,98 & 28,96 & 0,000 \\
$\mathrm{CV}$ & 1 & 255,3 & 255,32 & 25,58 & 0,000 \\
$\min$ & 1 & 307,6 & 307,60 & 30,82 & 0,000 \\
$\max$ & 1 & 0,1 & 0,068 & 0,01 & 0,935 \\
Erro & 35 & 349,3 & 9,980 & -- & -- \\
Total & 39 & 12830,0 & -- & -- & -- \\
\hline
\end{tabular}

* GL - graus de liberdade; $\mathrm{SQ}_{\mathrm{aj}}$ - soma de quadrados; $\mathrm{QM}_{\mathrm{aj}}$ - quadrados médios.

Da análise de variância do modelo de regressão (Tabela 5), o modelo mostra-se significativo, dado um coeficiente $\mathrm{R}_{\mathrm{aj}}^{2}=96,97 \%$, o que resulta em um erro próximo de $3 \%$. Constata-se que 03 (três) termos são significativos ( $\bar{x}, \mathrm{CV}, \min$ ) e apenas o termo referente ao máximo valor da resistência ( $\max$ ) não é significativo, o que possibilita excluí-lo do conjunto de coeficientes, por não influenciar nesta estimativa.

Admitindo, portanto, apenas os termos significativos para construir tal estimativa, adotam-se os três coeficientes significativos, conforme Equação 12, com os resultados da ANOVA expressos na Tabela 6.

$$
f_{\mathrm{t} 0, \mathrm{k}}^{\mathrm{Mod}}=\mathbf{2 0}, 28+\mathbf{0 , 4 0 9 9} \cdot \overline{\boldsymbol{x}}-\mathbf{0 , 8 2 4} \cdot \mathbf{C V}+\mathbf{0 , 3 6 9 4} \cdot \min \quad\left[\mathrm{R}_{\mathrm{aj}}^{2}=\mathbf{9 7}, \mathbf{0 5} \%\right]
$$


Tabela 6: Resultados da ANOVA para o modelo de regressão de acordo com a Equação 12.

\begin{tabular}{lccccc}
\hline FATOR & GL & $\mathrm{SQ}_{\mathrm{aj}}$ & $\mathrm{QM}_{\mathrm{aj}}$ & VALOR F & P-valor \\
\hline $\bar{x}$ & 1 & 980,0 & 980,01 & 100,99 & 0,000 \\
$\mathrm{CV}$ & 1 & 607,0 & 607,04 & 62,55 & 0,000 \\
$\min$ & 1 & 328,4 & 328,44 & 33,85 & 0,000 \\
Erro & 36 & 349,4 & 9,70 & -- & -- \\
Total & 39 & 12830,0 & -- & -- & -- \\
\hline
\end{tabular}

* GL - graus de liberdade; $\mathrm{SQ}_{\mathrm{aj}}$ - soma de quadrados; $\mathrm{QM}_{\mathrm{aj}}$ - quadrados médios.

Por fim, a Tabela 7 apresenta os resultados da ANOVA para avaliar a equivalência dos conjuntos de valores para $f_{\mathrm{t} 0, \mathrm{k}}$ (Equação 4) e $f_{\mathrm{t} 0, \mathrm{k}}^{\mathrm{Mod}}$ (Equação 12). Pelo P-valor inferior ao nível de significância (5\%), constata-se a não equivalência das médias dos dois grupos confrontados, ou seja, não possuem médias estatisticamente equivalentes entre si.

Tabela 7: Resultados da ANOVA para o conjunto de valores característicos da $\boldsymbol{f}_{\mathrm{t} 0, \mathbf{k}}$ e $\boldsymbol{f}_{\mathrm{t} 0, \mathbf{k}}^{\text {Mod }}$.

\begin{tabular}{lccccc}
\hline FONTE & GL & $\mathrm{SQ}_{\mathrm{aj}}$ & $\mathrm{QM}_{\mathrm{aj}}$ & VALOR F & P-valor \\
\hline Condição & 1 & 1762 & 1762,3 & 5,19 & 0,025 \\
Erro & 78 & 26493 & 339,70 & -- & -- \\
Total & 79 & 28255 & -- & -- & -- \\
\hline
\end{tabular}

* GL - graus de liberdade; $\mathrm{SQ}_{\mathrm{aj}}$ - soma de quadrados; $\mathrm{QM}_{\mathrm{aj}}$ - quadrados médios.

O teste de normalidade de Anderson-Darling resultou em um P-valor $<0,05$, assim como mostra a Figura 1, denotando a anormalidade da distribuição dos resíduos, indo de encontro ao resultado obtido pelo modelo da ANOVA, com um P-valor < 0,05 (Tabela 7).

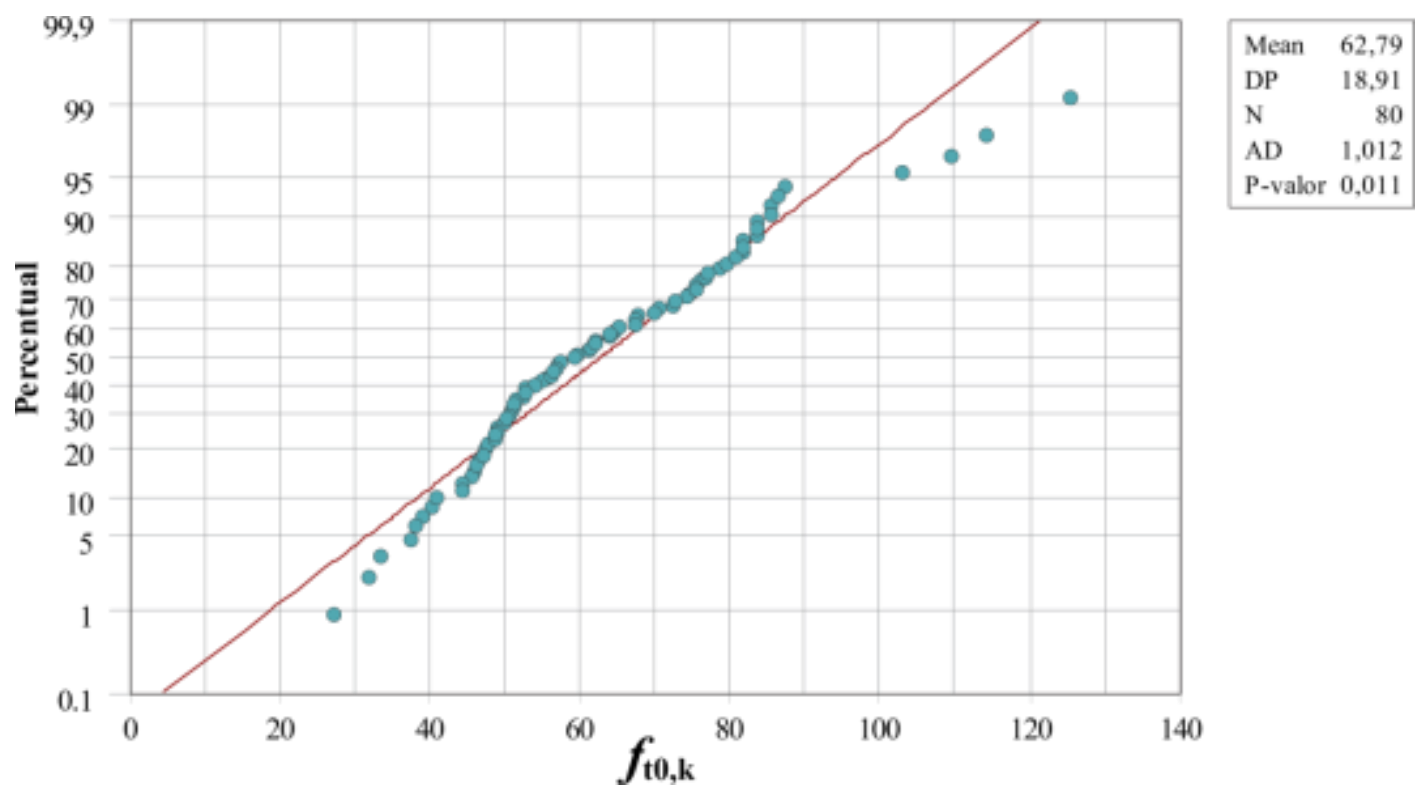

Figura 1: Resultados de validação da ANOVA: teste de normalidade de Anderson-Darling.

Em síntese, a Tabela 8 agrupa os valores característicos calculados, comparativamente, pela norma brasileira $\left(\boldsymbol{f}_{\mathbf{t} 0, \mathbf{k}}\right)$ (Tabela 2), pelas funções probabilísticas $\left(f_{\mathrm{t} 0, \mathrm{k}}^{\text {Prob }}\right)$ de melhor aderência (Tabela 3) e do modelo de regressão $\left(f_{\mathrm{t} 0, \mathrm{k}}^{\text {Mod }}\right)$ proposto neste trabalho (Equação 12). 
Tabela 8: Comparativo dos valores característicos para a tração via norma $\left(\boldsymbol{f}_{\mathbf{t} 0, \mathbf{k}}\right)$, função probabilística $\left(\boldsymbol{f}_{\mathbf{t} 0, \mathbf{k}}^{\text {Prob }}\right)$ e pelo modelo de regressão $\left(\boldsymbol{f}_{\mathbf{t} \mathbf{0}, \mathbf{k}}^{\text {Mod }}\right)$ aqui proposto.

\begin{tabular}{|c|c|c|c|c|c|c|c|c|c|}
\hline ID & POPULAR & $\begin{array}{c}\boldsymbol{f}_{\mathbf{t} 0, \mathbf{k}} \\
(\mathrm{Tab} .2)\end{array}$ & $\begin{array}{l}f_{\mathrm{t} 0, \mathrm{k}}^{\text {Prob }} \\
\text { (Tab. 3) }\end{array}$ & $\begin{array}{l}f_{\mathrm{t} 0, \mathrm{k}}^{\mathrm{Mod}} \\
(\mathrm{Eq} .12)\end{array}$ & ID & POPULAR & $\begin{array}{c}\boldsymbol{f}_{\mathbf{t} 0, \mathbf{k}} \\
\text { (Tab. 2) }\end{array}$ & $\begin{array}{l}f_{\mathrm{t} 0, \mathrm{k}}^{\text {Prob }} \\
\text { (Tab. 3) }\end{array}$ & $\begin{array}{l}f_{\mathrm{t} 0, \mathrm{k}}^{\mathrm{Mod}} \\
(\mathrm{Eq} .12)\end{array}$ \\
\hline 01 & Angelim-amargoso & 52,56 & 46,96 & 45,67 & 21 & Cutiúba & 75,22 & 61,01 & 61,76 \\
\hline 02 & Angelim-araroba & 48,63 & 44,31 & 42,23 & 22 & Garapa & 81,62 & 74,32 & 72,61 \\
\hline 03 & Angelim-ferro & 83,51 & 52,28 & 62,03 & 23 & Goiabão & 83,54 & 70,53 & 68,98 \\
\hline 04 & Angelim-pedra & 59,27 & 49,71 & 50,29 & 24 & Guaiçara & 80,59 & 78,56 & 76,39 \\
\hline 05 & Angelim-saia & 67,12 & 47,72 & 45,49 & 25 & Guajará & 83,43 & 76,13 & 74,06 \\
\hline 06 & Angelim-vermelho & 72,02 & 63,73 & 57,84 & 26 & Guarucaia & 63,98 & 56,77 & 56,81 \\
\hline 07 & Angico-branco & 53,94 & 50,73 & 54,95 & 27 & Itaúba & 72,60 & 57,41 & 60,23 \\
\hline 08 & Angico-preto & 76,65 & 55,92 & 58,30 & 28 & Jatobá & 125,29 & 113,99 & 109,95 \\
\hline 09 & Branquilho & 67,20 & 61,98 & 63,18 & 29 & Louro-preto & 61,36 & 55,46 & 57,59 \\
\hline 10 & Cafearana & 56,30 & 47,31 & 45,43 & 30 & Louro-verde & 69,87 & 67,47 & 68,07 \\
\hline 11 & Cambará-rosa & 31,79 & 27,17 & 25,16 & 31 & Maçaranduba & 109,40 & 102,75 & 102,49 \\
\hline 12 & Canafístula & 59,44 & 51,03 & 48,99 & 32 & Mandioqueira & 65,13 & 45,43 & 46,68 \\
\hline 13 & Castanheira & 61,95 & 54,80 & 56,27 & 33 & Oiticica-amarela & 74,23 & 64,55 & 62,84 \\
\hline 14 & Castelo & 86,42 & 81,49 & 80,34 & 34 & Oiuchu & 85,29 & 75,43 & 75,73 \\
\hline 15 & Catanudo & 48,79 & 37,88 & 32,66 & 35 & Parinari & 79,35 & 56,88 & 60,57 \\
\hline 16 & Cedro-amargo & 44,04 & 38,84 & 37,32 & 36 & Pau-óleo & 50,30 & 46,27 & 46,57 \\
\hline 17 & Cedro-doce & 48,55 & 45,91 & 45,45 & 37 & Piolho & 51,13 & 40,05 & 38,15 \\
\hline 18 & Cedroarana & 50,07 & 33,31 & 40,57 & 38 & Quarubarana & 40,63 & 37,40 & 37,55 \\
\hline 19 & Champanhe & 87,30 & 85,42 & 84,64 & 39 & Quina-rosa & 81,65 & 72,37 & 76,59 \\
\hline 20 & Copaíba & 52,67 & 48,82 & 48,97 & 40 & Rabo-de-arraia & 51,39 & 45,63 & 44,59 \\
\hline
\end{tabular}

Por fim, a Figura 2 ilustra os valores médios e os intervalos de confiança da média, para um nível de $95 \%$ de confiabilidade $(\mathrm{p}<0,05)$, dos valores da $f_{\mathrm{t} 0, \mathrm{k}}$ obtidos por ambas as formas de cálculo.

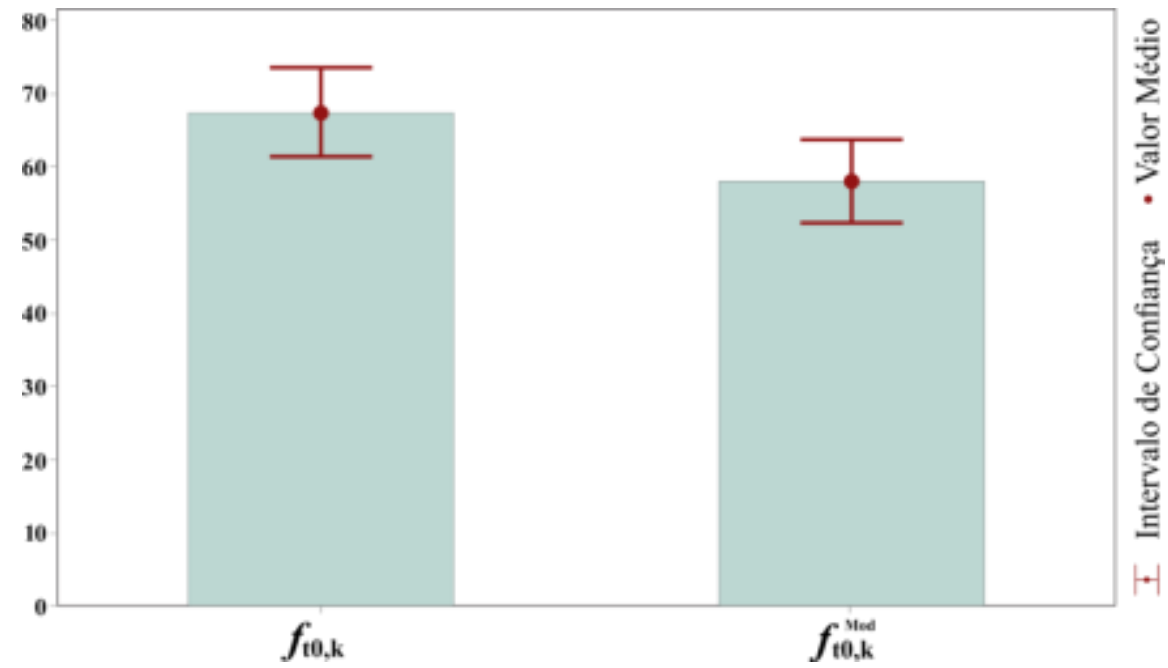

Figura 2: Valores médios e intervalos de confiança para a $f_{\mathrm{t} 0, \mathrm{k}}$ obtidos por ambos métodos de cálculo, respectivamente pela norma brasileira e pelo modelo aqui proposto (Equação 12).

A Figura 2 ajuda a elucidar a não equivalência dos dois conjuntos $\left(f_{\mathrm{t} 0, \mathrm{k}} \mathrm{e} f_{\mathrm{t} 0, \mathrm{k}}^{\mathrm{Mod}}\right)$, sendo que as médias atingiram: $f_{\mathrm{t} 0, \mathrm{k}}=67 \mathrm{MPa}$, enquanto que para $f_{\mathrm{t} 0, \mathrm{k}}^{\mathrm{Mod}}=58 \mathrm{MPa}$, respectivamente, para a norma (Equação 4) $\mathrm{e}$ para o modelo proposto neste trabalho (Equação 12).

\section{DISCUSSÃO}

Das 40 espécies aqui avaliadas (Tabela 1), muitas também foram objetos de estudos em outras pesquisas. Desde modo, cabe-se comparar as resistências $f_{\text {to }}$ (Tabela 4) com valores obtidos na literatura, a fim de cor- 
roborar com os valores encontrados neste trabalho.

Ao comparar as resistências $f_{\mathrm{t} 0}$ com os valores da NBR 7190 [1] (p. 90, Anexo E), que traz um total de 43 espécies do grupo das folhosas, sendo que 19 espécies são aqui avaliadas, verifica-se que todas estão dentro dos ICs aqui obtidos com os valores médios em sua maioria, próximos aos obtidos neste trabalho.

Podem-se comparar também os valores dispostos no estudo de Dias e Lahr [14], que avaliaram 40 espécies, dentre as quais 32 espécies são aqui avaliadas. Os valores médios da resistência $f_{\text {to }}$ obtidos pelos autores são congruentes com os valores alcançados neste trabalho, estando todos compreendidos no IC.

É possível ainda comparar alguns valores individuais para algumas das espécies estudadas neste trabalho. Os valores médios aqui encontrados para a $f_{\text {to }}$ se encontram no IC e podem ser equiparados aos obtidos por Lahr et al. [17] (Cambará-rosa (Erisma sp.) - $f_{\text {to }}=45 \mathrm{MPa}$ ), Aquino et al. [18] (Copaíba (Copaifera sp.) - $f_{\mathrm{t} 0}=71 \mathrm{MPa}$ ) e Christoforo et al. [6] (Castelo (Calycophyllum multiflorum) $-f_{\mathrm{t} 0}=104 \mathrm{MPa}$ ).

Destaca-se ainda o valor apresentado por Tenorio et al. [19] para duas populações de Cedro-amargo (Cedrela odorata), com o valor médio $f_{\text {to }}=40 \mathrm{MPa}$, sendo este inferior ao valor médio, mas no IC aqui calculado. Já a proposição de Lahr et al. [17] obteve um valor médio, para a espécie Jatobá (Hymenaea courbaril) extraída de três locais distintos, igual a $f_{\mathrm{t} 0}=154 \mathrm{MPa}$, valor este também compreendido dentro do IC obtido neste trabalho e próximo ao valor médio.

Outros autores avaliaram algumas das espécies aqui estudadas, porém, restritos às propriedades físicas: Itaúba (Mezilaurus itauba) [3]; Mandioqueira (Qualea sp) [5]; Cedro-amargo (Cedrela odorata) [19]; Cedrorana (Cedrelinga cateniformis) [20]; Garapa (Apuleia leiocarpa) [21] e Jatobá (Hymenaea sp.) [22].

\section{CONCLUSÕES}

Os resultados do modelo de regressão, proposto neste trabalho, alcançaram excelente precisão, mensurada pelo coeficiente $\mathrm{R}_{\mathrm{aj}}^{2}$ superior a $97 \%$. Ressalta-se a versatilidade no uso deste modelo para estimativa do valor característico da resistência $f_{\mathrm{t} 0, \mathrm{k}}$, uma vez que dependem apenas dos valores médio, mínimo e do coeficiente de variação, variáveis estas facilmente obtidas a partir do conjunto de dados experimentais ensaiados.

Ao confrontar o conjunto de valores característicos advindos do modelo apresentado neste trabalho, verifica-se a não equivalência estatística com o modelo proposto pela norma brasileira. Destaca-se o fato dos valores para $f_{\mathrm{t} 0, \mathrm{k}}^{\mathrm{Mod}}($ Equação 12$)$ atingirem resultados, na média, inferiores $(\approx 14 \%)$ aos obtidos pelas equações da norma e, portanto, a favor da segurança.

Tal questão reforça a importância da incorporação do modelo probabilístico aqui proposto à futura revisão da normativa para projetos estruturais de madeira no Brasil, tendo em vista a confiabilidade deste modelo aliada a facilidade de seu uso no que condiz a obtenção da resistência $f_{\text {to,k }}$, conduzida pelo conjunto de 40 espécies tropicais, que contemplam todas as classes de resistência das folhosas, grupo este imprescindível para o pré-dimensionamento estrutural em madeira.

\section{AGRADECIMENTOS}

Os autores agradecem o Conselho Nacional de Desenvolvimento Científico e Tecnológico (CNPq) pelo auxílio financeiro para o desenvolvimento deste trabalho.

\section{BIBLIOGRAFIA}

[1] ASSOCIAÇÃO BRASILEIRA DE NORMAS TÉCNICAS. NBR 7190: projeto de estruturas de madeira. Rio de Janeiro, 107 p., 1997.

[2] STEEGE, H., VAESSEN, R. W., LÓPEZ, D. C., et al., The discovery of the Amazonian tree flora with an update checklist of all known tree taxa, In: Scientific Reports, pp. 1-15, 2016.

[3] DA SILVA, F., HIGUCHI, N., NASCIMENTO, C. C., et al., "Nondestructive evaluation of hardness in tropical wood", Journal of Tropical Forest Science, v. 26, n. 1, pp. 69-74, 2014.

[4] CARREIRA, M. R., SEGUNDINHO, P. G. A., LAHR, F. A. R., et al., "Bending stiffness evaluation of teca and guajará lumber through tests of transverse and longitudinal vibration", Acta Scientiarum. Technology, v. 34, n. 1, pp. 27-32, 2012. 
[5] ALMEIDA, T. H., ALMEIDA, D. H., ARAUJO, V. A., et al., "Density as estimator of dimensional stability quantities of Brazilian tropical woods", BioResources, v. 12, n. 3, pp. 6579-6590, 2017.

[6] CHRISTOFORO, A. L., ARROYO, F. N., SILVA, D. A. L., et al., "Full characterization of Calycophyllum multiflorum wood specie", Journal of the Brazilian Association of Agricultural Engineering, v. 37, n. 4, pp. 637-643, 2017.

[7] LAHR, F. A. R., ARROYO, F. N., ALMEIDA, T. H., et al., "Full Characterization of Erisma uncinatum Warm. wood specie", International Journal of Materials Engineering, v. 6, n. 5, pp. 147-150, $2016 \mathrm{a}$.

[8] REIS, P. C. M. SOUZA, A. L., REIS, L. P., et al., "Artificial neural networks to estimate the physicalmechanical properties of Amazon second cutting cycle wood", Maderas. Ciencia y Tecnología, v. 20, n. 3, pp. 343-352, 2018.

[9] LOGSDON, N. B., JESUS, J. M. H., PENNA, J. E., "Avaliação dos estimadores da resistência característica à compressão paralela às fibras", Scientia Forestalis, v. 38, pp. 579-587, 2010.

[10] MATOS, G. S., MOLINA, J. C., "Resistência da madeira ao cisalhamento paralelo às fibras segundo as normas ABNT NBR 7190:1997 e ISO 13910:2005”, Revista Matéria, v. 21, n. 4, pp. 1069-1079, 2016.

[11] INTERNATIONAL STANDARD. ISO 13910: Structural timber - characteristic values of strengthgraded timber - sampling, full-size testing and evaluation. Suécia, 30 p., 2005.

[12] PINTO, E. M., ESPINOSA, M. M., CALIL JR., C., "Métodos para Determinação do Valor Característico da Resistência à Compressão Paralela às Fibras da Madeira", Madeira: Arquitetura e Engenharia, v. 5, n.14, pp. 1-6, 2004.

[13] ESPINOSA, M. M., CALIL JR., C., LAHR, F. A. R., "Métodos paramétricos e não-paramétricos para determinar o valor característico em resultados de ensaio de madeira", Scientia Forestalis, v. 66, pp. 76-83, 2004.

[14] DIAS, F. M., LAHR, F. A. R., “Análise das relações propostas pela norma brasileira NBR 7190 para caracterização simplificada da resistência de madeiras", In: Anais do Congresso Brasileiro de Engenharia e Ciência dos Materiais, São Pedro, SP, 2000.

[15] MINITAB, Minitab statistical software, http://www.minitab.com. Acessado em Agosto de 2018.

[16] WEERAHANDI, S., “ANOVA under unequal error variances”, International Biometric Society, v. 51, pp. 589-599, 1995.

[17] LAHR, F. A. R., CHRISTOFORO, A. L., SILVA, C. E. G., et al., "Avaliação de propriedades físicas e mecânicas de madeiras de Jatobá (Hymenaea stilbocarpa Hayne) com diferentes teores de umidade e extraídas de regiões distintas", Revista Árvore, v. 40, n. 1, pp. 147-154, 2016b.

[18] AQUINO, V. B. M., ALMEIDA, J. P. B., ALMEIDA, D. H., et al., "Physical and mechanical characterization of Copaifera sp. wood specie", International Journal of Materials Engineering, v. 8, n. 3, pp. 55-58, 2018.

[19] TENORIO, C., MOYA, R., "Evaluation of wood properties of four ages of Cedrela odorata trees growing in agroforestry systems with Theobroma cacao in Costa Rica", Agroforestry Systems, pp. 1-16, 2018.

[20] FERNANDES, N. C. L., VALLE, M. L. A., CALDERON, C. M. A., "Características físicas e anatômicas de Cedrela odorata L. e Cedrelinga cateniformis Ducke", Floresta e Ambiente, v. 25, n. 1, pp. 1$10,2018$.

[21] SORIANO, J., VEIGA, N. S., MARTINS, I. Z., "Wood density estimation using the sclerometric method”, European Journal of Wood and Wood Products, v. 73, pp. 753-758, 2015.

[22] TIAGO, P. V., ROSSI, A. A. B., CARPEJANI, A. A., et al., "Diversidade genética e estrutura populacional de Jatobá: uma espécie com potencial econômico para a Amazônia", Ciência Florestal, v. 28, n. 2, pp. 515-524, 2018.

\section{ORCID}

Anderson Renato Vobornik Wolenski

Rodrigo Guerra Peixoto

André Luís Christoforo

Francisco Antonio Rocco Lahr

Alfredo Manuel Pereira Geraldes Dias https://orcid.org/0000-0002-5709-1965

https://orcid.org/0000-0002-0163-211X

https://orcid.org/0000-0002-4066-080X

https://orcid.org/0000-0002-3510-8498

https://orcid.org/0000-0002-9752-1907 